\title{
Implementation and Development of Emergency Department Pharmacist-Driven Patient- Care Transitional Model: A Discussion of Our Experiences and Processes
}

Arinze Nkemdirim Okere, PharmD, MS, MBA, BCPS

College of Pharmacy and Pharmaceutical Sciences, Florida A\&M University

\begin{abstract}
Frequent preventable emergency department (ED) visits is an area of great concern among healthcare administrators. Although pharmacist interventions have been shown to reduce re-hospitalizations, studies demonstrating reduction in ED utilizations among the elderly are limited. Additionally, factors influencing readmissions in the ED are multifactorial. Hence, some healthcare systems struggle to reduce readmissions using pharmacy services. This has been a major issue facing care provided to the elderly in the ED. As healthcare care systems develops and implement a collaborative pharmacist and physician/mid-level provider comprehensive transitional model of care in the ED, the quality of care provided to the elderly will be enhanced which will ultimately translate to reduced inappropriate $E D$ visit and re-hospitalization with corresponding reduction in financial burden placed on both the patient, caregiver and the society. Therefore, the objective of this article is to discuss our process with implementing pharmacist-providers transitional model in the ED. The intent is to elucidate some pharmacist-intervention principles and a pharmacist-driven, patient-care transitional model that would provide direction for other healthcare systems to improve ED visits within their locality.
\end{abstract}

Keywords: Emergency Medicine, Pharmacist, Transition of Care, Clinical Pharmacy

\section{Introduction}

Preventable emergency department (ED) visits associated with chronic illness among the geriatric population (65 yrs +) are growing in number. In California, it was observed that ED visit rates were highest for renal disease $(49 \%)$, diseases of the blood and blood-forming organs (43.8\%), abdominal pain $(30 \%)$, other undefined symptoms $(27 \%)$, and hypertension (27\%). ${ }^{1}$ With $15 \%$ of all ED visits being made by patients at least 65 years and with the growing population of elderly population which is projected to increase from $14 \%$, in 2012 , to $20 \%$ in $2030{ }^{2}$ the incidence of ED visits associated with chronic disease across the states of the United States will continue to rise. The rising number of preventable ED visits among the elderly will not only financially impact the healthcare system but may adversely affect the individual patients. For example, in a matched cohort study, Nagurney et al observed an increased risk of functional decline by $17 \%$ among patients who visited the ED when compared to patients who did not. ${ }^{3}$ Although the result of the aforementioned observation is merely an association, it underscores the patient-related adverse outcomes associated with frequent ED visits among geriatric patients. It is noteworthy that among the elderly, medicationrelated problems are one of the significant reasons for increased ED utilizations. Therefore, it is reasonable to hypothesize that pharmacist intervention would improve ED

Corresponding author: Arinze Nkemdirim Okere, PharmD, MS, MBA, BCPS; Associate Professor

College of Pharmacy and Pharmaceutical Sciences

Florida A\&M University

1415 S Martin Luther King Jr Blvd, Tallahassee, FL 32301

Phone: 850-599-3109; Fax: 850-599-3347;

E-mail: preseoapc@msn.com utilizations. Although Pharmacist interventions have been shown to improve medication discrepancy, ${ }^{4}$ limited, randomized studies have demonstrated the impact of pharmacist interventions with the reduction of preventable ED use. Consequently, some healthcare systems struggle to reduce readmissions using pharmacy services. Hence, the objective of this article is to discuss our process with implementing pharmacist-providers transitional model in the ED with the intent of elucidating pharmacist intervention principles and a pharmacist-driven, patient-care transitional model that would provide direction for other healthcare systems to improve ED visits among the elderly within their locality.

\section{Our Experience with Implementation of Transitional Model of Care}

In 2009, we conducted a pilot study to evaluate the effectiveness of a pharmacist-led implementation of medication reconciliation services and medication therapy management, with student pharmacist assistance. ${ }^{5}$ The results of the study revealed a reduction in the frequency of combined ED visit and re-hospitalization among ED patients seen by student pharmacists under the direction of a pharmacist when compared to ED patients who followed the usual care (mean difference in number of visits: $25 \%, P=0.047) .{ }^{5}$ Although this pilot study was not a randomized clinical trial, the results of the pilot study was consistent with a randomized study published by Gillespie et al in Sweden. ${ }^{6}$ In Gillespie et al study, they observed a $16 \%$ reduction of combined ED visits and hospital readmissions among patients seen by pharmacists when compared to patients who did not (estimate 0.84\%; 95\% confidence interval $[\mathrm{Cl}], 0.72-0.99) .^{6}$ 
Following the results of our pilot study, we conducted a randomized control study in order to further evaluate the impact of pharmacist provisions of medication reconciliation and medication-therapy management on primary care visits, ED visits and urgent care visits. ${ }^{7}$ In this study, we observed an increased number of primary care visits (odds ratio [OR] 1.5; $95 \%[\mathrm{Cl}] 1.04-2.1$ ) and a reduction in the number of urgent care visits (OR $0.5 ; 95 \% \mathrm{Cl} 0.3-0.9$ ) among patients taking at least one chronic medication who were seen by a pharmacist compared to those who did not. ${ }^{7}$ However, no difference was observed in the frequency of ED visits between the two groups. We believed that the lack of statistical significant differences observed in the number of ED visits between the two groups was related to other quality improvement projects implemented by the health systems due to the roll out of the Affordable Care Act. Nonetheless, we concluded that the increase in the number of primary care visits and the reduction of urgent care visits was a positive sign of transitioning patients towards appropriate use of primary care services, which may advertently lead to an improvement in ED utilizations. ${ }^{7}$

Based on our positive experiences, we proposed that for the healthcare system to effectively improve preventable ED visits among elderly patients, ED pharmacist care model should integrate 4 fundamental intervention principles. These four fundamental intervention principles are as follows: 1.) Enhance the medication reconciliation process, 2.) Deprescribe (with respect to polypharmacy) 3.) Improve medication adherence, 4.) Improve patient medication education and counseling. The integration of the proposed intervention principles is based on the hypothesis that they are not independent of each other, and any deficiency in one of the intervention principles may lead to inappropriate ED utilization and adverse outcomes among the elderly. ${ }^{8-11}$ For example, though pharmacist provision of medication reconciliation alone has been shown to improve medication discrepancy, its sole application has not been shown to improve inappropriate ED utilizations. This is consistent with other principles when independently applied. Thus, highlighting the need to integrate the aforementioned four intervention principles in a pharmacist-care transitional model.

\section{Description of the Process Model}

Our patient care model (see Figure 1) systematically followed a stepwise approach and integrated into our ED triage process. It is noteworthy that some activities in the model, as will be described, either occurred simultaneously or concurrently. A description of the process is as follows:

Step 1. Patient's Profile Review: Upon presentation in the ED, Triage nurse alerts pharmacist for medication review. Five activities will occur concurrently at three different process levels as follows:
- Level 1: Pharmacist review of three important key elements: first, a review of past medical history; second, a history of current diseases and laboratory values (if available); third, review of past medication history ( $\mathrm{PMH}$ ) if available. The rationale for this interview or profile review is to correlate or identify any medication-related problem associated with history of present illness.

- Level 2: A trained Pharmacy technician or a student pharmacist will collect medication history.

- Level 3: ED Patient will be given a survey which has been psychometrically validated to proactively evaluate patient's medication-taking self-efficacy. This validated tool is known as the Okere-Renier Survey. ${ }^{12}$ The OkereRenier survey will be used by the pharmacist to proactively identify any deficiency in attitude or perception that may potentially lead to medication non-adherence. This survey can either be completed by the patient or the caregiver.

Step 2. Verification of patient's medication history: the patient's medication history will be compared with the medication history obtained from a single community pharmacy or a multiple community pharmacy (if the patient has more than one pharmacy). When the latter activity occurs, the ED provider will enter the patient's room to conduct a medical screening exam.

Step 3. Medication Therapy Management: The ED Physician/Provider and Pharmacist will quickly analyze information obtained. Critical information to be discussed are as follows: medication discrepancies, adherence patterns, drug-drug interactions, drug-disease interactions, potential drug-related adverse outcomes/side-effects, possible labs/imaging that could be obtained to support decisions and opportunities to improve care. Finally, a potential medication optimization related to the patient's comorbidities and deprescribing will be discussed.

Stage 4: Patient education/counseling: following step three, a pharmacist will provide medication information to the patient and a complete list of their accurate medication list. This will also be documented in the electronic health record (EHR). Information obtained from the Okere-Renier survey will be reviewed by the Pharmacist and will guide the pharmacist's approach in helping the patient improve his or her medicationtaking self-efficacy.

Stage 5: Discharge letter provided: in addition to a discharge letter being provided to the patient's primary care provider by the nurse, the pharmacist provides a short letter (usually less than a page) to summarize the patient's history of current illness, and to detail observed medication-related problems, the suggested approach to management and continued care after discharge. This is communicated to the primary care provider through electronic communication and included in the discharge letter sent by the provider. Finally, a copy of the letter written by the pharmacist is provided to the patient to aid with 
patient discussion with his or her primary provider. Most importantly, the content of this letter is also discussed with the patient prior to discharge. This latter activity is based on two rationales; first, the transfer of discharge letters (provided by ED providers) to the patient's outpatient provider is either not timely or lacks important information that would guide appropriate management and subsequently prevent readmissions. ${ }^{13,14}$ Secondly, discussing and providing the letter to the patients will empower them to discuss their care with their provider.

\section{Discussion}

Over the years, the primary objective of ED has evolved beyond treating critically ill patients to managing patients who have chronic diseases. With the elderly population being expected to increase exponentially in coming decades, the dire need to ameliorate inappropriate ED visit among the elderly population is widely acknowledged among healthcare organizations and policy makers.

As previously stated, many of the elderly that frequently visit the ED carry a high burden of chronic diseases, have multiple comorbidities and polypharmacy issues. ${ }^{15}$ The proposed transitional model of care process is based on a premise that the integration of pharmacists in the ED, as a frontline clinician, leads to an improved comprehensive medication review and minimize medication discrepancies prior to ED discharge. This will ultimately improve the transition of care and lead to better health outcomes. Thus, the adoption of this model will enhance healthcare system's ability to creatively use a interprofessional approach in the care of the elderly in the ED.

A unique characteristic of this model of care process is the ability to integrate several intervention principles such as medication reconciliation process, medication review that would lead to de-prescribing and optimization of medication therapy, helping patients improve medication adherence, provision of patient medication education and discharge counseling. As evident in our studies, ${ }^{5,7}$ it creates opportunities for the ED pharmacist to enhance patients' self-efficacy during ED visit which will ultimately lead to better health outcomes. Additionally, the proposed transition of model of care can enhance the algorithm to improve the safety and effectiveness of transition of care, as developed by Kessler et al. ${ }^{16}$ The aforementioned algorithm discussed the 5 step approach to transition of care in the ED, which involves minimizing interruptions, interprofessional approach, identification of high risk patients, identify the receiving patient and "closing the loop" which allowed time to address any questions. ${ }^{16}$ Thus, this model can easily be integrated in the algorithm to provide a true interprofessional approach to care.

Design and implementation of this proposed model is not without challenges. One of the noteworthy challenges was identifying the most productive time to have a pharmacist present in the ED as the institution, prior to implementation of the model, did not have a 24-hour ED pharmacy service. A collaboration and discussion between the ED team and the pharmacy team went a long way, not only to identify the right time period for maximal patient outreach but to overcome other challenges that the healthcare systems may encounter. Additionally, allocation of resources and staffing adjustment were potential barriers. The creative integration of either pharmacy students or trained pharmacy technician and/or post-graduate pharmacy resident in the model is key to overcoming the issue of resource allocation and staffing adjustment.

Despite these challenges, one unique advantage of this model is its simplicity to implement or to adapt irrespective of the type of healthcare system such as rural or urban, academic or nonacademic healthcare system. However, more pragmatic clinical studies will be needed to further evaluate the effectiveness of the proposed pharmacist transition of care model in improving health outcome, quality of care measures and its ease of implementation.

\section{Conclusion}

As previously stated, some healthcare systems struggle to reduce readmission-using pharmacy services. Nonetheless, reducing preventable readmissions is feasible when pharmacists and providers (including mid-level providers) collaboratively provide direct patient care adeptly. Efficient provisions of direct patient care in the emergency department will be dependent on the patient-care process model established. We propose that any patient-care model established should foster integration of interdisciplinary team members and the four fundamental intervention principles.

\section{Conflict of interest: none}

\section{References}

1. Hsia RY, Nath JB, Baker LC. California emergency department visit rates for medical conditions increased while visit rates for injuries fell, 2005-11. Health Aff (Millwood). 2015 Apr;34(4):621-6. doi: 10.1377/hlthaff.2014.0471

2. Ortman JM, Velkoff VA, Hogan H. An Aging Nation: The older population in the United States. U.S. Census Bureau, Current Population Reports, P251140. Washington, DC. 2014.

3. Nagurney JM, Fleischman W, Han L, Leo-Summers L, Allore HG, Gill TM. Emergency department visits without hospitalization are associated with functional decline in older persons. Ann Emerg Med. 2017 Apr; 69(4):426-433. doi: 10.1016/j.annemergmed.2016.09.018. 
4. Hohl CM, Partovi N, Ghement I, Wickham ME, et al. Impact of early inhospital medication review by clinic al pharmacists on health services utilization. PLUS One. 2017 Feb 13; 12(2):e0170495. doi: 10.1371/journal.pone.0170495.

5. Okere AN, Gessert CE, Renier CM, Swanoski M. Effects of medication reconciliation service provided by student pharmacists in a tertiary care emergency department. Innov Pharm. 2013;4(2):1-9 [article 13]. Available at:

http://www.pharmacy.umn.edu/innovations/prod/gr oups/cop/@pub/@ cop/@innov/documents/article/cop_article_440661. pdf. Accessed January 19, 2015.

6. Gillespie U, Alassaad A, Henrohn D, Garmo $H$, Hammarlund-Udenaes $M$, Toss $H$, Kettis-Lindblad A, Melhus H, Mörlin C.A comprehensive pharmacist intervention to reduce morbidity in patients 80 years or older: a randomized controlled trial. Arch Intern Med. 2009 May 11; 169(9):894-900. doi: 10.1001/archinternmed.2009.71.

7. Okere AN, Renier CM, Tomsche JJ. Evaluation of the influence of a pharmacist-led patient-centered medication therapy management and reconciliation service in collaboration with emergency department physicians. J Manag Care Spec Pharm. 2015 Apr; 21(4):298-306.

8. Roulet L, Ballereau F, Hardouin JB, Chiffoleau A, Potel $G$, Asseray N. Adverse drug event nonrecognition in emergency departments: an exploratory study on factors related to patients and drugs. J Emerg Med. 2014 Jun; 46(6):857-64. doi: 10.1016/j.jemermed.2013.11.124.

9. Heaton PC, Tundia NL, Luder HR. U.S. emergency departments visits resulting from poor medication adherence: 2005-07. J Am Pharm Assoc (2003). 2013 Sep-Oct;53(5):513-9. doi: 10.1331/JAPhA.2013.12213.

10. Malhotra S, Karan RS, Pandhi P, Jain S.

Drug related medical emergencies in the elderly: role of adverse drug reactions and non-compliance. Postgrad Med J. 2001 Nov; 77(913):703-7.
11. Cheen $\mathrm{MHH}$, Goon $\mathrm{CP}$, Ong WC, Lim PS, Wan $\mathrm{CN}$, Leong $\mathrm{MY}$, Khee GY. Evaluation of a care transition program with pharmacist-provided, home-based medication review for elderly Singaporeans at high risk of readmissions. Int J Qual Health Care. 2017 Apr 1; 29(2):200-205. doi: 10.1093/intqhc/mzw150.

12. Okere AN, Renier CM, Morse J. Development and validation of a survey to assess patient-perceived medication knowledge and confidence in medication use. J Nurs Meas. 2014; 22(1):120-34.

13. Kripalani S, LeFevre F, Phillips CO, Williams MV, Basaviah P, Baker DW. Deficits in communication and information transfer between hospital-based and primary care physicians: implications for patient safety and continuity of care. JAMA. 2007 Feb 28; 297(8):831-41.

14. Kattel S, Manning DM, Erwin PJ, Wood H, Kashiwagi DT, Murad MH. Information Transfer at Hospital Discharge: A Systematic Review. J Patient Saf. 2016 Jan 7. [Epub ahead of print]

15. Downing A, Wilson R. Older people's use of Accident and Emergency services. Age Ageing. 2005 Jan;34(1):24-30. Epub 2004 Oct 20

16. Kessler C, Shakeel F, Hern HG, Jones JS, Comes J, Kulstad C, Gallahue FA, Burns BD, Knapp BJ, Gang M, Davenport M, Osborne B, Velez LI. An algorithm for transition of care in the emergency department. Acad Emerg Med. 2013 Jun;20(6):605-10. doi: 10.1111/acem.12153. 
Figure 1

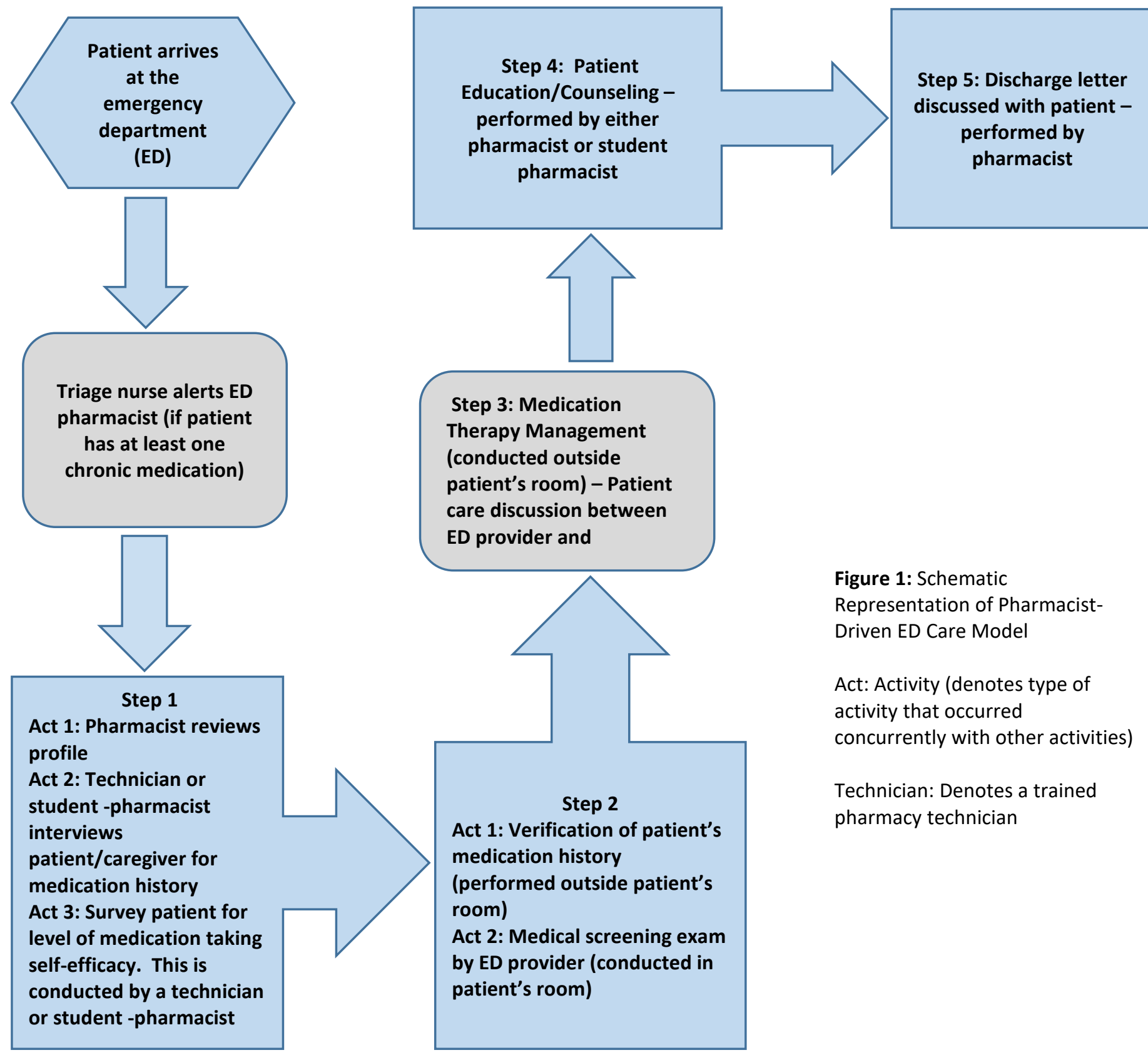

